\title{
Absence of Fragmentation in Two-Dimensional Bose-Einstein Condensation
}

\author{
Juan Pablo Fernández and William J. Mullin \\ Department of Physics, Hasbrouck Laboratory \\ University of Massachusetts, Amherst, MA 01003, USA
}

\begin{abstract}
We investigate the possibility that the BEC-like phenomena recently detected on two-dimensional finite trapped systems consist of fragmented condensates. We derive and diagonalize the one-body density matrix of a two-dimensional isotropically trapped Bose gas at finite temperature. For the ideal gas, the procedure reproduces the exact harmonic-oscillator eigenfunctions and the Bose distribution. We use a new collocation-minimization method to study the interacting gas in the Hartree-Fock approximation and obtain a groundstate wavefunction and condensate fraction consistent with those obtained by other methods. The populations of the next few eigenstates increase at the expense of the ground state but continue to be negligible; this supports the conclusion that two-dimensional BEC is into a single state.
\end{abstract}

PACS numbers: 03.75.Hh, 05.30.Jp, 05.70.Fh, 32.80.Pj

Bose-Einstein condensation (BEC) cannot occur at finite temperature in a strictly two-dimensional trapped interacting gas in the thermodynamic limit 1 On the other hand, phenomena resembling BEC have been detected by experiments ${ }^{2}$ and Monte Carlo simulations ${ }^{3}$ on two-dimensional finite trapped systems. These could consist of "fragmented" condensates, $\frac{4}{4}$ in which atoms avalanche macroscopically not just into the ground state but into a finite set of low-energy states. Fragmented condensation has been studied in the context of spin-1 systems, double potential wells, and rotating systems with attractive interparticle interactions (the bibliography of Ref. 4 contains a complete set of references); moreover, a mean-field analysis of a homogeneous three-dimensional Bose gas with repulsive interactions ${ }^{5}$ rules out the possibility of fragmentation in such a system. To our knowledge, however, nobody has yet treated trapped $2 \mathrm{D}$ systems, and that is the topic 


\section{J. P. Fernández and W. J. Mullin}

of this work. In what follows we will derive and diagonalize the one-body density matrix - whose eigenvectors and eigenvalues respectively correspond to the one-body wavefunctions of the system and their populations - of a two-dimensional isotropically trapped Bose gas at finite temperature using the Hartree-Fock-Bogoliubov equations in the Hartree-Fock approximation. The results we obtain are consistent with condensation into a single state.

The generalized criterion for Bose-Einstein condensation, first introduced by Penrose and Onsager,$\left[\frac{6}{}\right.$ relies on the properties of the one-body density matrix (OBDM) of a system of identical bosons. This quantity, defined by the ensemble average $n\left(\mathbf{r}, \mathbf{r}^{\prime}\right) \equiv\left\langle\Psi^{\dagger}(\mathbf{r}) \Psi\left(\mathbf{r}^{\prime}\right)\right\rangle$, where $\Psi$ is a many-body field operator, has the following properties for the case of a trapped-and hence inhomogeneous - two-dimensional gas: $(i)$ its diagonal, $n(\mathbf{r}) \equiv n(\mathbf{r}, \mathbf{r})$, provides the spatial density of the system; $(i i)$ its trace is the total number of atoms in the gas: $\int d^{2} r n(\mathbf{r})=N$; (iii) field operators are Hermitian, and can hence be expanded in the form $\Psi(\mathbf{r})=\sum_{k} \phi_{k}(\mathbf{r}) b_{k}$; the annihilation (and creation) operators obey the standard commutation relations, while the functions $\phi_{k}(\mathbf{r})$ form part of a complete orthonormal set. Use of the condition $\left\langle b_{j}^{\dagger} b_{k}\right\rangle=f_{j} \delta_{j k}$, where $f_{j}$ can be interpreted as the number of atoms in the $j$ th state, yields

$$
\tilde{n}\left(\mathbf{x}, \mathbf{x}^{\prime}\right)=\sum_{k} f_{k} \phi_{k}^{*}(\mathbf{x}) \phi_{k}\left(\mathbf{x}^{\prime}\right)
$$

Note that we have expressed the variables using the length and energy scales set by the trap: $\mathbf{x} \equiv \mathbf{r}(m \omega / \hbar)^{1 / 2}$ is the position vector, and the density matrix and wavefunctions have been made dimensionless; in particular, $\tilde{n} \equiv$ $\hbar n / m \omega$. It is straightforward to show that (11) implies

$$
\int d^{2} x^{\prime} \tilde{n}\left(\mathbf{x}, \mathbf{x}^{\prime}\right) \phi_{j}\left(\mathbf{x}^{\prime}\right)=f_{j} \phi_{j}(\mathbf{x})
$$

This expression can be interpreted as an eigenvalue equation for the operator $\int d^{2} x^{\prime} \tilde{n}\left(\mathbf{x}, \mathbf{x}^{\prime}\right)$; the resulting eigenvectors are the one-body eigenstates and the corresponding eigenvalues are their occupation numbers. In the particular case of the ideal gas, these populations are dictated by the Bose-Einstein distribution, which prescribes a condensation into the oscillator ground state at low but finite temperatures. ${ }^{1}$ The Penrose-Onsager criterion is a generalization of this result: A system is said to have a Bose-Einstein condensate if its OBDM has a single eigenvalue of order $N$-identified with the condensate population - while the rest are orders of magnitude smaller; if more than one eigenvalue is of order $N$, the system exhibits a fragmented condensate; if all of the eigenvalues are of order 1 , the system is uncondensed.

The density matrix (11), however, is not amenable to numerical diagonalization as it stands, since it is a four-dimensional array with continuous 


\section{Absence of Fragmentation in Two-Dimensional BEC}

indices. In order to turn it into a diagonalizable matrix we must approximate the integrals by discrete sums and average over all but two of these dimensions; this last condition makes the process feasible only for isotropic traps. In what follows we will diagonalize the averaged matrix

$$
\left.\bar{n}\left(x, x^{\prime}\right) \equiv \frac{1}{2 \pi} \int_{0}^{2 \pi} d \varphi^{\prime} \tilde{n}\left(\mathbf{x}, \mathbf{x}^{\prime}\right)\right|_{\varphi=0} .
$$

The Hartree-Fock-Bogoliubov equations have been used with remarkable success in the study of Bose-Einstein condensation, and have been applied to the study of two-dimensional systems. ${ }^{[7}$ The model assumes an interparticle interaction $V\left(\mathbf{x}_{1}-\mathbf{x}_{2}\right)=\gamma \delta^{(2)}\left(\mathbf{x}_{1}-\mathbf{x}_{2}\right)$, with $\gamma$ positive for repulsive interactions, and decomposes the field operator $\Psi(\mathbf{x})$ in the form

$$
\Psi=\langle\Psi\rangle+\tilde{\psi} \equiv \tilde{\Phi}+\tilde{\psi},
$$

where the ensemble average $\tilde{\Phi}$ is a real macroscopic wavefunction whose square is the condensate density $\tilde{n}_{0}$ and the fluctuations about this average correspond to a thermal cloud of density $\tilde{n}^{\prime}=\left\langle\tilde{\psi}^{\dagger} \tilde{\psi}\right\rangle$. When we insert (44) into the many-body grand canonical Hamiltonian and neglect the "anomalous" averages $\left\langle\tilde{\psi}^{\dagger} \tilde{\psi}^{\dagger}\right\rangle$ and $\langle\tilde{\psi} \tilde{\psi}\rangle$, we obtain coupled expressions for the condensate and the noncondensate. The condensate is described by the Gross-Pitaevskil equation,

$$
\tilde{\nabla}^{2} \tilde{\Phi}+\left(\tilde{\mu}-x^{2}\right) \tilde{\Phi}-\gamma\left(\tilde{n}_{0}+2 \tilde{n}^{\prime}\right) \tilde{\Phi}=0,
$$

and the thermal density in the Hartree-Fock approximation ${ }^{8}$ is given by

$$
\tilde{n}^{\prime}(\mathbf{x})=-\frac{1}{4 \pi \tilde{\beta}} \log \left(1-\exp \left[-\tilde{\beta}\left(x^{2}+2 \gamma \tilde{n}-\tilde{\mu}\right)\right]\right),
$$

where we have introduced the inverse temperature $\tilde{\beta} \equiv \hbar \omega \beta / 2$ and the chemical potential $\tilde{\mu} \equiv 2 \mu / \hbar \omega$. Equations (5) and (6), together with the imposition that $N=\int d^{2} x\left(\tilde{n}_{0}+\tilde{n}^{\prime}\right)$, form a self-consistent set of equations that can be solved to study the complete thermodynamics of the trapped system at any temperature, with no adjustable parameters, given $N$ and $\gamma$.

This identification also gives us an expression for the off-diagonal elements of the density matrix. From the definition

$$
\tilde{n}\left(\mathbf{x}, \mathbf{x}^{\prime}\right) \equiv\left\langle\Psi^{\dagger}(\mathbf{x}) \Psi\left(\mathbf{x}^{\prime}\right)\right\rangle=\tilde{\Phi}^{*}(\mathbf{x}) \tilde{\Phi}\left(\mathbf{x}^{\prime}\right)+\left\langle\tilde{\psi}^{\dagger}(\mathbf{x}) \tilde{\psi}\left(\mathbf{x}^{\prime}\right)\right\rangle,
$$

where we have once again neglected the anomalous averages, we obtain ${ }^{8}$

$$
\begin{array}{r}
\tilde{n}\left(\mathbf{x}, \mathbf{x}^{\prime}\right)=\tilde{\Phi}(\mathbf{x}) \tilde{\Phi}\left(\mathbf{x}^{\prime}\right)+\frac{1}{4 \pi \tilde{\beta}} \sum_{\ell=1}^{\infty} \frac{1}{\ell} \exp \left(-\frac{1}{4 \tilde{\beta} \ell}\left(x^{2}+x^{\prime 2}-2 \mathbf{x} \cdot \mathbf{x}^{\prime}\right)\right. \\
\left.-\ell \tilde{\beta}\left[\frac{1}{2}\left(x^{2}+x^{\prime 2}\right)+\gamma\left(\tilde{n}(\mathbf{x})+\tilde{n}\left(\mathbf{x}^{\prime}\right)\right)-\tilde{\mu}\right]\right),
\end{array}
$$




\section{J. P. Fernández and W. J. Mullin}

which results from neglecting the position-momentum commutator and using the free-particle result $\left\langle\mathbf{x}\left|e^{-\tilde{\beta} \kappa^{2}}\right| \mathbf{x}^{\prime}\right\rangle=e^{-\left(\mathbf{x}-\mathbf{x}^{\prime}\right)^{2} / 4 \tilde{\beta}} / 4 \pi \tilde{\beta}$.

To solve the Hartree-Fock equations in the presence of a condensate we follow a self-consistent procedure: Initially, we assume that only the condensate is present and solve Eq. (15) with $\tilde{n}^{\prime}=0$. The wavefunction and chemical potential that result are fed into the Hartree-Fock expression for the density (6), which, when integrated, yields also a value for the noncondensate population $N^{\prime}$; one can then readjust the condensate population and solve the Gross-Pitaevskil equation that results. The process is iterated until the chemical potential and the populations stop changing. To solve the nonlinear eigenvalue problem (5) we directly minimize the functiona 9

$$
J[\tilde{\Phi}]=\int d^{2} x\left[(\tilde{\nabla} \Phi)^{2}+\Phi\left(x^{2}+2 \gamma \tilde{n}^{\prime}(\mathbf{x})\right) \Phi+\frac{\gamma}{2} \Phi^{4}\right]
$$

subject to the constraint $\int d^{2} x \Phi^{2}=1 .\left(\tilde{\Phi}=\sqrt{N_{0}} \Phi\right.$.) We first set a grid of $n$ fixed abscissas $x_{j}^{(n)}$ that represent the radial coördinate $x$; in terms of the ordinates $f_{j} \equiv \Phi\left(x_{j}^{(n)}\right), J[\tilde{\Phi}]$ becomes a multivariate function, $J=$ $J\left[\left(f_{1}, \ldots, f_{n}\right)^{T}\right] \equiv J[\mathbf{f}]$, that can be minimized using standard optimization routines. The resulting function is known at the $x_{j}^{(n)}$ and can be evaluated everywhere else by interpolation.

For each evaluation of $J$ we need to be able to calculate both derivatives and integrals of functions that we know only at a few points. We have developed a method that uses as grid points the zeros of $L_{n}$, the $n$th Laguerre polynomial; the calculation of $J[\mathbf{f}]$ is reduced to the matrix-vector product

$$
J[\mathbf{f}]=\frac{1}{\Delta} \sum_{j=1}^{n}\left(2 \pi x_{j}^{(n)}\right)\left((\mathbf{D} \mathbf{f})_{j}^{2}+\left(x_{j}^{(n)} f_{j}\right)^{2}+2 \gamma \tilde{n}^{\prime}\left(x_{j}^{(n)}\right) f_{j}^{2}+\frac{\gamma f_{j}^{4}}{2 \Delta}\right) w_{j}^{(n)} e^{x_{j}^{(n)}},
$$

where $\Delta \equiv \sum_{j=1}^{n}\left(2 \pi x_{j}^{(n)}\right) f_{j}^{2} w_{j}^{(n)} e^{x_{j}^{(n)}}$ is a normalization factor. The $w_{j}^{(n)} e^{x_{j}^{(n)}}$ are Gauss-Laguerre weights, including the inverse of the weighting function, 10 evaluated at the grid points: $w_{j}^{(n)}=\left(L_{n}^{\prime}\left(x_{j}^{(n)}\right)\right)^{-2} / x_{j}^{(n)}$. Finally, D is a differentiation matrix, $\frac{11}{11}$ whose elements are given by

$$
D_{i j}=\frac{L_{n}^{\prime \prime}\left(x_{j}^{(n)}\right)}{2 L_{n}^{\prime}\left(x_{j}^{(n)}\right)} \delta_{i j}+\frac{L_{n}^{\prime}\left(x_{i}^{(n)}\right)}{L_{n}^{\prime}\left(x_{j}^{(n)}\right)} \frac{1-\delta_{i j}}{x_{i}^{(n)}-x_{j}^{(n)}} .
$$

Now, the change of variables $x_{j}^{(n)} \rightarrow x_{j}^{(n)} / b$ with any positive $b$ will map the semi-infinite interval onto itself provided that we also rescale $\exp \left(x_{j}^{(n)}\right) \rightarrow$ $\exp \left(b x_{j}^{(n)}\right), w_{j}^{(n)} \rightarrow w_{j}^{(n)} / b$, and $\mathrm{D} \rightarrow b \mathrm{D}$. This free parameter can be tweaked by hand in order to optimize the accuracy of the minimization process. The 

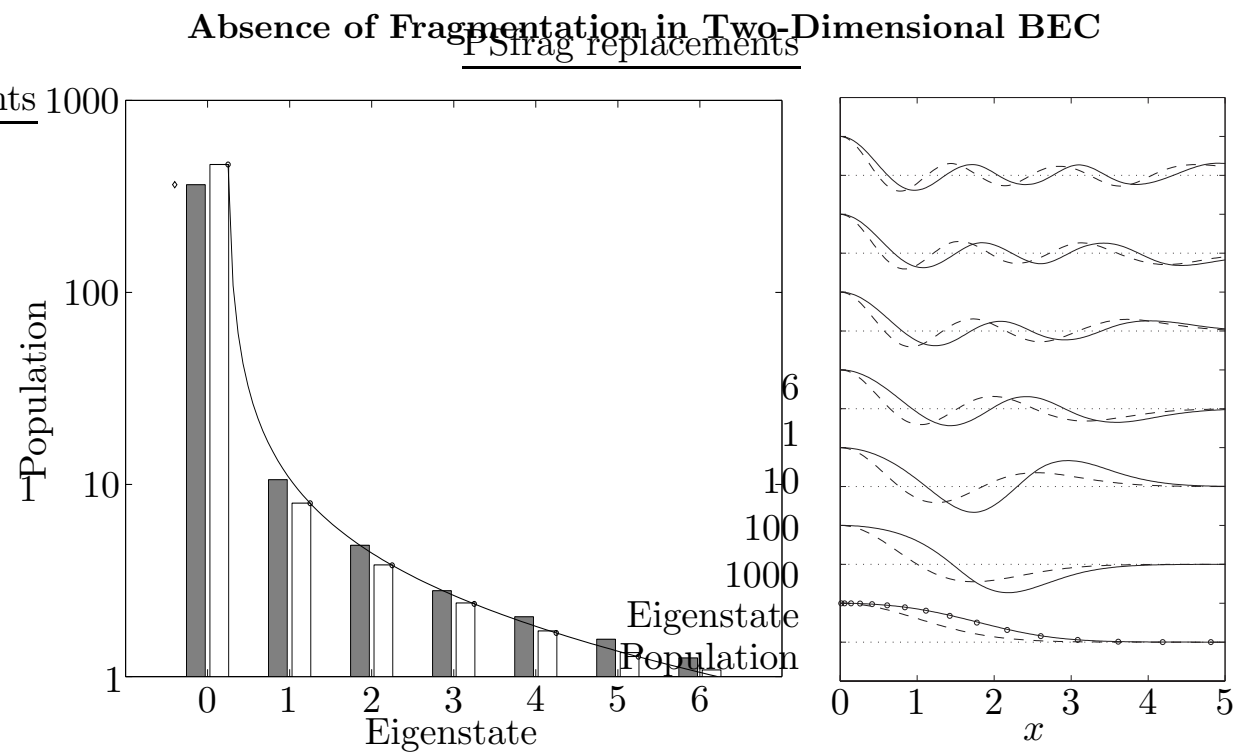

Fig. 1. Eigenvalues (left panel) and eigenvectors (right panel) of the onebody density matrix of a two-dimensional interacting trapped Bose gas and the equivalent ideal gas.

method just described yields self-consistent density profiles in mere seconds and is quite robust.

The left panel of Fig. 11 shows the seven lowest eigenvalues of the angleaveraged OBDM corresponding to an isotropic two-dimensional interacting Bose gas in the Hartree-Fock approximation (gray bars) compared to those of the equivalent ideal gas (white bars). In both cases $N=1000$ and $T=0.7 T_{c}$, where $T_{c}$ is the ideal-gas transition temperature ${ }^{1}$ The interaction parameter, $\gamma=8 \pi \times 0.0043$, is arbitrary in $2 \mathrm{D}$ but corresponds to a $3 \mathrm{D}$ gas of rubidium atoms $\frac{9}{9}$ In both cases we calculated the matrix on a $30 \times 30$ Laguerre grid.

The ideal-gas results have been obtained by means of an exact expression for the OBDM,

$$
\tilde{n}\left(\mathbf{x}, \mathbf{x}^{\prime}\right)=\frac{1}{\pi} \sum_{\ell=1}^{\infty} \frac{e^{\ell \tilde{\beta}(\tilde{\mu}-2)}}{1-e^{-4 \ell \tilde{\beta}}} e^{-\frac{1}{2} \operatorname{csch} 2 \ell \tilde{\beta}\left(\left(x^{2}+x^{\prime 2}\right) \cosh 2 \ell \tilde{\beta}-2 \mathbf{x} \cdot \mathbf{x}^{\prime}\right)},
$$

which is found by direct evaluation of (11) and can be proved analytically to obey (2) with populations prescribed by the Bose-Einstein distribution, $f_{k}=\left(\exp \left(\tilde{\beta}\left[\epsilon_{k}-\tilde{\mu}\right]\right)-1\right)^{-1}$, where, in our system of units, $\epsilon_{k}=2(2 k+1)$; these values are represented in the figure by open circles, and the straight line depicts the distribution for continuous $k$. The good agreement between analytic and numerical results provides a useful check on our algorithms.

The interacting-gas results clearly show the depletion of the condensate due to interactions and the complementary increase of the excited occupation 


\section{J. P. Fernández and W. J. Mullin}

numbers. However, the ground-state population is still orders of magnitude greater than that of any other state (note that the scale on the $y$-axis is logarithmic). In this particular case, for example, the condensate number decreases from 463 to 363 but the population of the first excited state increases only from 8 to 11 . Finally, the open diamond at the left shows the condensate number yielded by the self-consistent procedure.

The right panel of the figure exhibits the corresponding eigenfunctions, which have been normalized to be unity at $x=0$. The solid lines represent the (spline-interpolated) interacting eigenfunctions; the ideal-gas eigenfunctions (dashed lines) have been calculated numerically but are indistinguishable from the analytic wavefunctions $\phi_{n}(\mathbf{x})=e^{-x^{2} / 2} L_{n}\left(x^{2}\right) / \sqrt{\pi}$. The open circles at the bottom show the Gauss-Laguerre points where the functions are known exactly and depict the condensate wavefunction that resulted from the self-consistent procedure. The wavefunctions decrease in value and are flattened at the center of the trap; on the other hand, the radius of the system becomes larger, and both condensate and noncondensate are affected.

Our results lead us to the conclusion that, in finite 2D systems, and to this level of approximation, there is a phenomenon resembling BEC into a single state. This conclusion is supported further by Monte Carlo simulations that we have performed on squeezed 3D gases and whose results we intend to publish in the future.

\section{REFERENCES}

1. W. J. Mullin, J. Low Temp. Phys. 106, 615 (1997).

2. A. Görlitz et al., Phys. Rev. Lett. 87, 130402 (2001); D. Rychtarik, B. Engeser, H.-C. Nägerl, and R. Grimm, Phys. Rev. Lett. 92, 173003 (2004).

3. S. D. Heinrichs and W. J. Mullin, J. Low Temp. Phys. 113, 231 (1998); ibid. 114, 571 (1999).

4. W. J. Mullin, M. Holzmann, and F. Laloë, J. Low Temp. Phys. 121, 263 (2000).

5. P. Nozières, in Bose-Einstein Condensation, edited by A. Griffin, D. W. Snoke, and S. Stringari (Cambridge University Press, Cambridge, 1993), p. 15.

6. O. Penrose and L. Onsager, Phys. Rev. 104, 576 (1956).

7. C. Gies, B. P. van Zyl, S. A. Morgan, and D. A. W. Hutchinson, Phys. Rev. A 69, 023616 (2004); L. Pricoupenko, Phys. Rev. A 70, 013601 (2004).

8. M. Holzmann and Y. Castin, Eur. Phys. J. D 7, 425 (1999).

9. W. Krauth, Phys. Rev. Lett. 77, 3695 (1996).

10. W. H. Press, S. A. Teukolsky, W. A. Vetterling, and B. P. Flannery, Numerical Recipes in C (Cambridge University Press, Cambridge, 1992), 2nd ed.

11. J. A. C. Weideman and S. C. Reddy, ACM Trans. of Math. Software 26, 465 (2000). 